\title{
Leveraging Implementation Science to Improve Delivery of Oncologic Reconstructive Surgery
}

\author{
Nicholas L. Berlin, MD, MPH ${ }^{1,2}$, and Anaeze C. Offodile II, MD, MPH ${ }^{3,4,5}$ \\ ${ }^{1}$ Section of Plastic Surgery, University of Michigan, Ann Arbor, MI; ${ }^{2}$ National Clinician Scholars Program, University of \\ Michigan Institute for Healthcare Policy and Innovation, Ann Arbor, MI; ${ }^{3}$ Department of Plastic Surgery, The University \\ of Texas MD Anderson Cancer Center, Houston, TX ; ${ }^{4}$ Baker Institute for Public Policy, Rice University, Houston, TX; \\ ${ }^{5}$ Department of Health Services Research, The University of Texas MD Anderson Cancer Center, Houston, TX
}

A consequence of the rapid technological advancements and sub-specialization of oncology practice we have witnessed during the past two decades is the increased complexity of care delivery. Patients and caregivers now must navigate the care continuum of screening, treatment, survivorship, and symptom palliation with a diverse group of providers across multiple care settings. As a result, effective co-coordination of care for patients with a diagnosis of cancer represents is one of the greatest challenges facing contemporary health care systems.

Recognizing the complexity of coordinating stakeholders, both the American Society of Clinical Oncology and the European Society for Medical Oncology have promoted multidisciplinary care as the gold standard and framework for providing optimal cancer treatment. ${ }^{1}$ The improved overall survival and patient outcomes associated with multidisciplinary cancer care teams validate this position. ${ }^{2-5}$ Although varying definitions exist for multidisciplinary care, the central tenets are bidirectional communication across disciplines and a shared understanding of a patient-specific comprehensive treatment plan.

The recent article by Retrouvey et al. ${ }^{6}$ draws attention to a crucial but often overlooked topic in the spectrum of cancer care (i.e., the role and utility of reconstructive plastic surgeons). The value of reconstructive surgery in

(C) Society of Surgical Oncology 2020

First Received: 31 March 2020;

Published Online: 12 April 2020

A. C. Offodile II, MD, MPH

e-mail: aoffodile2@gmail.com; acoffodile@mdanderson.org oncology is increasingly gaining acceptance due to improved oncologic survival and an accompanying shift in focus to functional and quality-of-life outcomes for cancer patients. Currently, many health systems still struggle to identify and operationalize a role for plastic surgeons in an interprofessional collaborative model for oncology care.

Breast reconstruction represents an ideal use case for unpacking this issue given the annual incidence and policy relevance of breast cancer. Through the years, improved psychosocial outcomes (e.g., body image and psychosocial well-being) and quality of life (e.g., physical and sexual well-being) without adverse sequela of oncologic outcomes have been associated with post-mastectomy reconstruction. $^{7-9}$ These gains are heightened for patients with oropharyngeal cancers and extremity sarcomas, with modern reconstructive techniques (e.g., microsurgery, three-dimensional modeling, and customized implants) enabling modest to significant functional recovery (ambulation, speech, and smile). Finally, reconstructive surgery through shared decision-making, allows for more personalized treatment decisions for cancer patients.

Patient access to post-mastectomy reconstruction has received considerable attention during the past few decades. Despite mandated insurance coverage in the United States through the Women's Health and Cancer Rights Act, socioeconomic, geographic, and health care barriers continue to limit access to these procedures. ${ }^{10-15}$ At a local health care level, discussions about reconstruction during the initial consultation with the breast cancer surgeon have been shown to have significant impact on the likelihood of patients receiving reconstruction. ${ }^{16}$ From this context, Retrouvey et al. ${ }^{6}$ sought to explore the perceived role of multidisciplinary collaboration in the delivery of these 
procedures. Through qualitative interviews with 30 participants (22 physicians and 8 administrators), the authors explored the attitudes, beliefs, and values of key stakeholders in the provision of breast cancer care to patients from six centers across Ontario, Canada. The authors should be commended for this important study, which offers a nuanced understanding of the local contextual factors that have an impact on access to these procedures.

The major findings of the study underscore important challenges to interprofessional collaboration and care coordination. At a physician level, the lack of role clarity, the absence of an explicitly defined leader, and the varying perceptions of the usefulness of breast reconstruction have discouraged collaboration and rendered its delivery more difficult. The authors also discovered inconsistent views concerning the role of plastic surgeons on the oncologic team. Although plastic surgeons considered themselves members of the oncologic team, their colleagues generally disagreed because reconstructive procedures have not been considered cancer-related treatments. Most general surgeons thought it was their responsibility to discuss breast reconstruction as an option with their patients, and administrators appeared to play a key role in the delivery of breast reconstruction conditional on their values and goals.

In the designing of multi-level strategies to improve access to breast reconstruction, the findings from this study might be best served by the framework of the adapted model for the Tailored Implementation in Chronic Diseases (TICD). ${ }^{17-19}$ This model provides a number of potential levers to improve interprofessional collaboration at the local level (i.e., incentives, organization of professional interactions, resources, and capacity for organizational change).

In addressing professional interactions, strategies should focus on improving communication and leveraging these channels with the greatest influence. The National Accreditation Program for Breast Centers has endorsed a standard mandating that surgical oncologists offer a referral for plastic surgery at the time of breast cancer diagnosis. Implementation of this standard in local health care settings must entail regular audits of referral volume and the reversal of any identified disincentives for referral to plastic surgery. Furthermore, resources such as decisionsupport tools, patient education materials, and establishment of a longitudinal patient navigation program are essential.

Organizational changes may require allocation or reallocation of authority to make referral decisions. It may be helpful to appoint an implementation leader, such as an administrator or clinical champion, to define clear guidelines for deciding who is accountable for initially discussing the option of breast reconstruction with patients considering mastectomy. The referral process should be transparent, with consideration of individual feedback loops to providers.

The role of health policy in promoting access to breast reconstruction cannot be overstated, although it is uneven because poor access for racial minorities and economically disadvantaged women persist. ${ }^{11,12,15}$ Recognizing barriers posed by communication and referral patterns in access to breast reconstruction, the state of New York enacted Public Health (NY PBH) Law 2803-O in 2010, which mandates that surgeons communicate about reconstructive surgery with patients undergoing mastectomy. Initial policy evaluations have shown that this approach likely reduced racial and ethnic disparities in access to these procedures ${ }^{20}$.

In conclusion, the challenges posed by care coordination for cancer patients demand thoughtful solutions that leverage principles of implementation science and, in some cases, health policy. Given advances in modern-day reconstructive techniques, nearly all patients with oncologic defects may benefit from consultation and coordination with plastic surgery. Improving interprofessional collaboration between oncologic teams and plastic surgeons represents another important opportunity to advance patient-centered cancer care.

ACKNOWLEDGMENT Nicholas L. Berlin receives funding from the Department of Veterans Affairs that supports his role as a National Clinician Scholar.

DISCLOSURE There are no conflicts of interest.

\section{REFERENCES}

1. American Society of Clinical Oncology. ASCO-ESMO consensus statement on quality cancer care. J Clin Oncol. 2006;24:3498-9.

2. Hong NJ, Wright FC, Gagliardi AR, Paszat LF. Examining the potential relationship between multidisciplinary cancer care and patient survival: an international literature review. J Surg Oncol. 2010;102:125-34.

3. Friedland PL, Bozic B, Dewar J, Kuan R, Meyer C, Phillips M. Impact of multidisciplinary team management in head and neck cancer patients. Br J Cancer. 2011;104:1246-8. .

4. Tsai WC, Kung PT, Wang ST, Huang KH, Liu SA. Beneficial impact of multidisciplinary team management on the survival in different stages of oral cavity cancer patients: results of a nationwide cohort study in Taiwan. Oral Oncol. 2015;51:105-11.

5. Yee-Ting Cheng T, Szumacher E, Di Prospero L. Breast and prostate cancer patient perspectives and perceptions of interprofessional collaboration during cancer treatment: a pilot study. $J$ Med Imaging Radiat Sci. 2014;45:373-81.

6. Retrouvey HZT, Gagliardi A, Baxter N, Webster F. How ineffective interprofessional collaboration affects the delivery of breast reconstruction to breast cancer patients: a qualitative study. Ann Surg Oncol. 2020. https://doi.org/10.1245/s10434-020-0846 3-0.

7. Pusic AL, Matros E, Fine N, et al. Patient-reported outcomes 1 year after immediate breast reconstruction: results of the 
Mastectomy Reconstruction Outcomes Consortium Study. J Clin Oncol. 2017;35:2499-506.

8. Santosa KB, Qi J, Kim HM, Hamill JB, Wilkins EG, Pusic AL. long-term patient-reported outcomes in postmastectomy breast reconstruction. JAMA Surg. 2018;153:891-9.

9. Nelson JA, Allen RJ Jr, Polanco T, et al. Long-term patientreported outcomes following postmastectomy breast reconstruction: an 8-year examination of 3268 patients. Ann Surg. 2019;270:473-83.

10. Jagsi R, Jiang J, Momoh AO, et al. Trends and variation in use of breast reconstruction in patients with breast cancer undergoing mastectomy in the United States. J Clin Oncol. 2014;32:919-26.

11. Offodile AC II, Tsai TC, Wenger JB, Guo L. Racial disparities in the type of postmastectomy reconstruction chosen. J Surg Res. 2015;195:368-76. .

12. Alderman AK, Hawley ST, Janz NK, et al. Racial and ethnic disparities in the use of postmastectomy breast reconstruction: results from a population-based study. $J$ Clin Oncol. 2009;27:5325-30.

13. Alderman AK, Atisha D, Streu R, et al. Patterns and correlates of postmastectomy breast reconstruction by U.S. plastic surgeons: results from a national survey. Plast Reconstr Surg. 2011;127:1796-803.

14. Berlin NL, Wilkins EG, Alderman AK. Addressing continued disparities in access to breast reconstruction on the 20th anniversary of the Women's Health and Cancer Rights Act. JAMA Surg. 2018;153:603-4.
15. Tseng JF, Kronowitz SJ, Sun CC, et al. The effect of ethnicity on immediate reconstruction rates after mastectomy for breast cancer. Cancer. 2004;101:1514-23.

16. Greenberg CC, Schneider EC, Lipsitz SR, et al. Do variations in provider discussions explain socioeconomic disparities in postmastectomy breast reconstruction? $J$ Am Coll Surg. 2008;206:605-15.

17. Flottorp SA, Oxman AD, Krause J, et al. A checklist for identifying determinants of practice: a systematic review and synthesis of frameworks and taxonomies of factors that prevent or enable improvements in healthcare professional practice. Implement Sci. 2013;8:35. DOI:10.1186/1748-5908-1188-1135.

18. Damschroder LJ, Aron DC, Keith RE, Kirsh SR, Alexander JA, Lowery JC. Fostering implementation of health services research findings into practice: a consolidated framework for advancing implementation science. Implement Sci. 2009;4:50. DOI:10.1186/ 1748-5908-1184-1150.

19. Wensing M. The Tailored Implementation in Chronic Diseases (TICD) project: introduction and main findings. Implement Sci. 2017;12:5. DOI: 10.1186/s13012-13016-10536-x.

20. Mahmoudi E, Lu Y, Metz AK, Momoh AO, Chung KC. Association of a policy mandating physician-patient communication with racial/ethnic disparities in postmastectomy breast reconstruction. JAMA Surg. 2017;152:775-83.

Publisher's Note Springer Nature remains neutral with regard to jurisdictional claims in published maps and institutional affiliations. 